\title{
THE EXISTENCE OF COMBINATORIAL FORMULAE FOR CHARACTERISTIC CLASSES \\ BY
}

\author{
NORMAN LEVITT AND COLIN ROURKE
}

\begin{abstract}
Given a characteristic class on a locally ordered combinatorial manifold $M$ there exists a cocycle which represents the class on $M$ and is locally defined, i.e. its value on $\sigma \in M$ depends only on the ordered star st $(\sigma, M)$. For rational classes the dependence on order disappears. There is also a locally defined cycle which carries the dual homology class.
\end{abstract}

For some time it has been known that there is a simple combinatorial representation for the homology duals of the Stiefel-Whitney classes of a combinatorial manifold (Whitney [8], cf. Cheeger [1], Halperin and Toledo [2]). It is natural to ask whether there is an analogous result for other characteristic classes. For instance, can one give a simple combinatorial formula for the Pontrjagin classes or for their homology duals? What is being sought is a formula which depends only on the local structure of the combinatorial manifold $K$ (as a simplicial complex).

In this paper we prove a theoretical result. We establish that formulae of this type exist for all characteristic classes and for their homology duals. But the method of proof makes it extremely difficult to actually give such a formula explicitly. Our formulae depend, in general, on local ordering of the complex, but for rational classes (such as the rational Pontrjagin classes) this dependence disappears. Miller [4] has shown that the rational characteristic numbers of $K$ are in fact the only numerical invariants of $K$ which admit formulae in terms of the local (unordered) structure of $K$. Thus, for a general characteristic class, some other datum such as our local ordering is necessary.

One corollary to the existence of local formulae is that any manifold which can be triangulated so that the links of $q$-simplexes admit orientation reversing simplicial isomorphisms has all cohomology rational characteristic classes of dimension $n-q$ zero.

Cheeger has communicated to us that he has a proof of existence of

Received by the editors April 1, 1977. Based in part on a section of a manuscript by the first author, received by the editors on July 22, 1976.

AMS (MOS) subject classifications (1970). Primary 55F40; Secondary 55F60, 55C50, 57D20.

Key words and phrases. Combinatorial formula, characteristic class, Pontrjagin class, local formula, classifying block bundle. 
formulae for real Pontrjagin classes of smoothly triangulated smooth manifolds. His method of proof is quite different from ours.

The first author would like to acknowledge a helpful conversation with $\mathbf{R}$. MacPherson, and we would refer the reader to MacPherson's Bourbaki seminar [3] on Gelfand's work towards an explicit local formula for the first Pontrjagin class.

A word about the method of proof. We shall construct a classifying space $\mathbf{Q}_{n}$ which is natural for ordered triangulated block bundles. (I.e. there is a unique classifying map for such a block bundle.) Given an ordered combinatorial manifold $K$, then its tangent bundle $\tau_{K}$ is such a block bundle and hence we have a unique map $f: K \rightarrow \mathbf{Q}_{n}$ which depends only on the local structure of $K$. Our "combinatorial formulae" then come from pulling back representatives on $\mathbf{Q}_{n}$.

1. Definitions. A total ordering or partial ordering of a simplicial complex $K$ is a total or partial ordering on the vertices.

A local ordering of $K$ is a partial ordering on $K$ which restricts to a total order on each (closed) star st $(\sigma, K)$ for $\sigma \in K$. We regard two local orderings as equivalent if the total order on each star is the same.

An s-ball (ordered simplicial ball) is an ordered complex $K$ s.t. $|K|$ is a ball.

An s-cell complex is a partially ordered simplicial complex $K$ together with a family of subcomplexes $\left\{L_{i}\right\}$ such that

(1) each $L_{i}$ is totally ordered and is an $s$-ball of some dimension;

(2) the polyhedra $|K|,\left\{\left|L_{i}\right|\right\}$ satisfy the usual conditions for a cell complex (Rourke and Sanderson [5, p. 3]).

Another way of thinking of an s-cell complex is that it is a cell complex with a partially ordered triangulation wherein each cell is triangulated by a totally ordered subcomplex.

An isomorphism $h: K_{1} \rightarrow K_{2}$ between $s$-cell complexes is a simplicial isomorphism which is also an isomorphism of cell complexes and preserves the total ordering in each cell.

For instance if $K$ is a locally ordered combinatorial manifold then the dual complex $K^{*}$ is an $s$-cell complex, since the local ordering on $K$ induces a partial ordering on the first derived $K^{(1)}$, such that each cell of $K^{*}$ is totally ordered. Equivalent local orderings on $K$ give isomorphic dual complexes.

An s-block bundle $\xi / K$ of fibre dimension $n$, where $K$ is an $s$-cell complex, is a partially ordered complex $Q \supset K$ together with totally ordered subcomplexes $\left\{R_{i}\right\}$ (one for each cell $\left.L_{i} \subset K\right)$ such that each $R_{i}$ is an s-ball and $|Q|$, $\left\{\left|R_{i}\right|\right\}$ form an $n$-block bundle over the underlying cell complex $|K|,\left\{\left|L_{i}\right|\right\}$. An isomorphism of $s$-block bundles is a simplicial isomorphism which is also an isomorphism of block bundles and preserves the total order on each block.

For example if $K \subset L$ are locally ordered combinatorial manifolds and $|K|$ 
is locally flatly embedded in $|L|$, then the normal bundle $\nu(K, L) / K^{*}$ (see $[5$, p. 1]) is an $s$-block bundle, since the blocks are cells of $L^{*}$. Equivalent local orderings give rise to isomorphic normal bundles.

Now suppose $K$ is a locally ordered combinatorial manifold, then the simplicial product $K \times K$ is also locally ordered and has the diagonal $\Delta K$ as a subcomplex. Then the tangent bundle of $K, \tau K$, which is defined to be $\nu(\Delta K, K \times K)$ is again an s-block bundle, and equivalent local orderings of $K$ give rise to isomorphic tangent bundles.

2. The classifying bundle. We are goint to construct a universal $s$-block bundle (of fibre dimension $n$ ). We need to use a "semi-simplicial" category appropriate to $s$-block bundles.

An s-cell is an s-cell complex with a single top dimensional cell of which the other cells are all faces. The model category $\delta$-cell has for objects isomorphism classes of $s$-cells, and for morphisms face inclusions. An $\delta$-cellset is a contravariant function from $\delta$-cell to the category of sets. An $\delta$-cell-set $Q$ possesses a geometric realisation $|Q|$ which is defined by gluing together representatives for the cells, via the face maps, in the usual way [6, $p$. 325].

Observe that $\Delta$ the model category for $\Delta$-sets (with objects the standard ordered $n$-simplexes, for each $n$, and morphisms order preserving face inclusions) is a full subcategory of $\delta$-cell. Hence given a $\Delta$-set $Q$ we can define an $\delta$-cell-set by mapping the other objects to the empty set. We denote this $\mathcal{S}$-cell-set by $Q$ as well. Thus we have a functor

$$
\{\Delta \text {-sets }\} \rightarrow\{\delta \text {-cell-sets }\} \text {. }
$$

There is also a functor which goes the other way. Given an $\mathcal{\delta}$-cell-set $Q$ then the realisation $|Q|$ is made of $s$-cells glued together. Now each $s$-cell is made of ordered simplexes so $|Q|$ has a natural subdivision which makes it the realisation of a $\Delta$-set $Q_{\Delta}$. I.e. $|Q|=\left|Q_{\Delta}\right|$. Notice that if $Q$ is already a $\Delta$-set then $Q_{\Delta}=Q$.

It now follows from the $\Delta$-approximation theorem $[6,5.1]$ that there is an approximation theorem for $\mathcal{S}$-cell-sets and that the homotopy category of $\{\delta$-cell-sets $\}$ is naturally equivalent to the homotopy category of CW complexes. There is also a good theory of "Kan" $\delta$-cell-sets, which we will not need to use.

We now define the classifying space for $s$-block bundles of fibre dimension $n$.

The $\mathcal{S}$-cell-set $\mathbf{Q}_{n}$ associates to an $s$-cell $K$ the set of isomorphism classes of $s$-block bundles with base $K$ of fibre dimension $n$. Face maps in $\mathbf{Q}_{n}$ are defined "by restriction" (cf. [5, p. 10]).

Given any $s$-block bundle $\xi / K$ there is a canonical $\delta$-cell-map $i_{\xi}: K \rightarrow \mathbf{Q}_{n}$ 
given by associating to the cell $L_{i} \subset K$ the class of the block $R_{i}$ in $\xi$ and given any $\delta$-cell-map $f: K \rightarrow \mathbf{Q}_{n}$ we can construct an $s$-block bundle $\xi / K$ by taking representatives for the images $f\left(L_{i}\right)$ for each $i$ and gluing them by means of the face maps in $\mathbf{Q}_{n}$ which correspond under $f$ to the face inclusions of $K$. (Notice that any such map has a unique geometric realisation since it is an order-preserving simplicial isomorphism.)

In fact, we can define the notion of $s$-block bundle over an $\delta$-cell-set as in $[7, \S 2]$ and glue the cells of $\mathbf{Q}_{n}$ together to form the classifying bundle $\gamma / \mathbf{Q}_{n}$. Then the construction of $\xi$ in the last paragraph is just the pull-back $f^{*}(\gamma)$.

PROPOSITION. $\gamma / \mathbf{Q}_{n}$ is a classifying bundle for $n$-dimensional block bundles. In particular $\left|\mathbf{Q}_{n}\right|$ has the homotopy type of $\left|B \widetilde{P L_{n}}\right|$.

PRoof. We need to show that concordance classes of $n$-block bundles over a polyhedron $P$ correspond via the pull back of $\gamma$ to homotopy classes of maps $P \rightarrow\left|Q_{n}\right|$ and then the result follows by a formal argument.

Given $\xi / P$ we can form an $s$-block bundle by triangulating all the blocks and cells and ordering arbitrarily. There is then the natural map $i_{\xi}: P \rightarrow\left|\mathbf{Q}_{n}\right|$; Notice that $i_{\xi}^{*}(\gamma)=\xi$. Given two such $s$-block bundles, we form an extension over $P \times I$ by putting a common subdivision at level $\left\{\frac{1}{2}\right\}$ and starring at levels $\left\{\frac{1}{4}\right\}$ and $\left\{\frac{3}{4}\right\}$ and then ordering compatibly with the ends. Thus we have a function

$$
\{\text { concordance classes of block bundles over } P\} \rightarrow\left[P,\left|Q_{n}\right|\right] \text {. }
$$

Now given a map $P \rightarrow\left|\mathbf{Q}_{n}\right|$ we can approximate by a $\Delta$-map and pull back $\gamma$ to obtain a block bundle, and by the relative approximation theorem the result is unique up to concordance. These two processes are inverse, and the proposition is proved.

3. The existence of combinatorial formulae. For simplicity we deal first with unoriented characteristic classes.

A class $\rho$ in $H^{*}\left(\left|B \widetilde{P L}_{n}\right|, G\right)$, where $G$ is an abelian group, is an $G$-characteristic class for $n$-dimensional block bundles. Using the homotopy equivalence $\left|B \widetilde{P L}_{n}\right| \simeq\left|\mathbf{Q}_{n}\right|$ of the proposition, we can represent $\rho$ by a cochain on $\left|\mathbf{Q}_{n}\right|$. Here we remark that $\left|\mathbf{Q}_{n}\right|$ has two natural cell structures.

(A) we can regard the semicells as cells,

(B) we can regard the simplexes in the semicells as cells (i.e. we are using the natural cell structure of $\left.\left|\left(\mathbf{Q}_{n}\right)_{\Delta}\right|\right)$.

Choose representatives $\rho_{A}$ and $\rho_{B}$ for $\rho$ as cochains corresponding to each cell structure. Then given an $s$-block bundle $\xi / K$, the canonical map $f$ : $K \rightarrow \mathbf{Q}_{n}$ gives us two cochains $f^{*} \rho_{A}$ and $f^{*} \rho_{B}$ which carry the characteristic class $\rho$ on $\xi$. 
Now let $K$ be a combinatorial manifold. By the characteristic class $\rho$ on $K$ we mean the characteristic class $\rho$ on the tangent block bundle of $K$.

THEOREM 1. Let $K$ be a locally ordered combinatorial manifold. Then there is a cochain $\rho_{K}$ on $K^{(1)}$ (with coefficients in $G$ ) which represents $\rho$ on $K$ and which is locally defined. In other words its value on a simplex $\sigma \in K^{(1)}$ depends only on the isomorphism class of the ordered complex $\operatorname{st}(\tau, K)$ (where $\sigma$ starts with $\hat{\tau}$ ).

Proof. Let $f: K^{*} \rightarrow \mathbf{Q}_{n}$ classify the $s$-block bundle $\tau_{K}$. Then $f$ induces $\rho_{K}=f^{*} \rho_{B}$ a simplicial cochain on $K^{(1)}$ which represents the characteristic class $\rho$ on $K$. It remains to observe that $f$, and hence $\rho_{K}$, depends only on the local structure of $K$ as a locally ordered combinatorial manifold.

COROLlary 1. Let $\rho$ be a rational characteristic class then there is a representative $\bar{\rho}_{K}$ for $\rho$ on $K^{(1)}$ which depends only on the local (unordered) structure of $K$.

Proof. Let $\rho_{K_{1}}, \ldots, \rho_{K_{r}}$ be the representatives given by all possible local orders on $K$. Define $\bar{\rho}_{K}$ to be the average of $\rho_{K_{1}}, \ldots, \rho_{K}$. Then $\rho_{K}$ has value on $\sigma \in K^{(1)}$ the average of the values corresponding to the possible orders of st $(\tau, K)$. Hence $\bar{\rho}_{K}$ depends only on the local unordered structure of $K$.

THEOREM 2. Given $K$ oriented, there is a simplicial chain $\rho_{K}^{*}$ on $K$ which represents the dual to $\rho$ on $K$ and which is locally defined, i.e. it is of the form $\Sigma g(\sigma) \sigma$ where $g(\sigma)$ depends only on the (ordered) complex st $(\sigma, K)$.

Proof. Consider $f^{*} \rho_{A}$. This is a cochain on $K^{*}$ which represents $\rho$ on $K$. Its dual is the required chain $\rho^{*}{ }_{K}$ on $K$.

COROLlaRY 2. Rational homology characteristic classes have representatives which are locally defined and independent of order.

Proof. Average, as in the proof of Corollary 1.

Remarks. (1) We can think of one of our "combinatorial formulae" as a function.

$$
\left\{\begin{array}{l}
\text { isomorphism classes } \\
\text { of stars st }(\tau, K)
\end{array}\right\} \rightarrow\left\{\begin{array}{l}
\text { cochains on } \\
\operatorname{st}(\tau, K)^{(1)}
\end{array}\right\}
$$

and the cochains fit together to give the required cocycle. For the homology representatives the situation is rather more simple. We in fact have a function.

$$
g:\{\text { isomorphism classes of stars }\} \rightarrow G
$$

such that $\Sigma g(\tau) \tau \mid \tau \in K_{q}^{(1)}$ is the required representative.

(2) In the context of Theorem 2 we may want to deal with oriented classes which are not pullbacks of unoriented classes. (Note that stable rational 
classes, e.g. Pontrjagin or $L$-classes, do arise from unoriented classes.) Consider the orientation double cover

$$
\begin{aligned}
& \mathbf{R}_{n} \simeq B S \widetilde{P L_{n}} \\
& \downarrow \\
& \mathbf{Q}_{n} \simeq B \widetilde{P L_{n}}
\end{aligned}
$$

Then the orientation of $K$ determines a lift $\tilde{f}$ of the classifying map $f: K \rightarrow \mathbf{Q}_{n}$ in $\mathbf{R}_{n}$. If we now represent our oriented class on $\mathbf{R}_{n}$ as a cochain $\rho$ then $\tilde{f}^{*}(\rho)$ is a representative on $K^{(1)}$ or $K^{*}$ which depends both on the local structure of $K$ and its orientation.

(3) Our results work equally well for homology manifolds (either rational or integral): The idea is to do all the constructions in the homology category, i.e. replace the notion of $s$-ball by $s$-homology-ball, $s$-cell-complex by $s$-homology-cell-complex, and block-bundle by homology block-bundle. We then get a space $h \mathbf{Q}_{n}$ which classifies homology block-bundles. The arguments of this section then give local formulae for arbitrary characteristic classes (of homology manifolds), which are independent of order for rational characteristic classes (e.g. Pontrjagin classes).

(4) It remains to prove the corollary stated at the beginning of the paper. Suppose $|K|=M$, that $\sigma \in K$ is a $q$-simplex and that $i: \operatorname{lk}(\sigma, K) \rightarrow \mathrm{k}(\sigma, K)$ is an orientation reversing simplicial isomorphism. Then $i$ induces an isomorphism $i^{*}: \sigma^{*} \rightarrow \sigma^{*}$. We use the representation of $\rho$ on the dual complex $K^{*}$. Recall that the value on $\sigma^{*}$ is obtained by averaging $\rho_{K}^{*}\left(\sigma^{*}\right)$ over all possible local orders for $K$. Now if $\mu$ is an order for $\sigma^{*}$ then $\mu \circ i$ is another order, and the corresponding values of $\rho_{K}^{*}$ are the same but opposite in sign (both correspond to mapping to the same cell of $\mathbf{Q}_{n}$ and the one map is the other map composed with $i$, which reverses orientation). It is not difficult to see that the local orderings can be paired off in this way and hence the average is zero on $\sigma^{*}$. So if all the $q$-links admit such orientation reversing isomorphisms, then the cochain representing $\rho$ is identically zero. Note that the proof does not use orientability of $M$.

\section{REFERENCES}

1. J. Cheeger, Topology of manifolds, J. C. Cantrell and C. H. Edwards, Jr., Eds., Markham, Chicago, Ill., 1970, pp. 470-471.

2. S. Halperin and D. Toledo, Stiefel-Whitney homology classes, Ann. of Math. (2) 96 (1972), 511-525.

3. R. MacPherson, Sem. Bourbaki 29 (1976-77), No. 497.

4. E. Miller, Ph.D. Thesis, Harvard Univ., Cambridge, Mass., 1973.

5. C. P. Rourke and B. J. Sanderson, Block bundles. I, Ann. of Math. (2) 87 (1968), 1-28.

6. ___ A-sets. I, Homotopy theory, Quart. J. Math. Oxford Ser. 22 (1971), 321-338.

7. $\longrightarrow \Delta$-sets. II, Block bundles block fibrations, Quart. J. Math. Oxford Ser. 22 (1971), $465-485$. 
8. H. Whitney, On the theory of sphere bundles, Proc. Nat. Acad. Sci. U.S.A. 26 (1940), 148-153.

Department of Mathematics, Rutgers Universtty, New Brunswick, New JerSey 08903 (Current address of Norman Levitt)

Department of Mathematics, UNIVERStTy OF WARWICK, WarWick, ENGLAND

Faculty of Mathematics, The Open University, Milton Keynes, England MK7 6AA (Current address of Colin Rourke) 